\title{
Identification by MALDI-TOF MS and Antibiotic Resistance of Riemerella anatipestifer, Isolated from a Clinical Case in Commercial Broiler Chickens
}

\author{
Athina Tzora ${ }^{1, *}$, Stylianos Skoufos ${ }^{1}$, Eleftherios Bonos ${ }^{1} \mathbb{(}$, , Konstantina Fotou ${ }^{1}$, Achilleas Karamoutsios ${ }^{1}{ }^{1}$, \\ Aikaterini Nelli ${ }^{1}$, Ilias Giannenas ${ }^{2}$, Anastasios Tsinas ${ }^{1}$ and Ioannis Skoufos ${ }^{1}$ \\ 1 Laboratory of Animal Health, Food Hygiene and Quality, Department of Agriculture, School of Agriculture, \\ University of Ioannina, Kostakioi Artas, 47100 Arta, Greece; steliosskoufos@gmail.com (S.S.); \\ ebonos@uoi.gr (E.B.); kfotou@uoi.gr (K.F.); a.karamoutsios@uoi.gr (A.K.); k.nelli@uoi.gr (A.N.); \\ actsinas@uoi.gr (A.T.); jskoufos@uoi.gr (I.S.) \\ 2 Laboratory of Nutrition, School of Veterinary Medicine, Faculty of Health Sciences, Aristotle University of \\ Thessaloniki, 54124 Thessaloniki, Greece; igiannenas@vet.auth.gr \\ * Correspondence: tzora@uoi.gr
}

check for updates

Citation: Tzora, A.; Skoufos, S.; Bonos, E.; Fotou, K.; Karamoutsios, A.; Nelli, A.; Giannenas, I.; Tsinas, A.; Skoufos, I. Identification by MALDI-TOF MS and Antibiotic Resistance of Riemerella anatipestifer, Isolated from a Clinical Case in Commercial Broiler Chickens. Vet. Sci. 2021, 8, 29. https://doi.org/ $10.3390 /$ vetsci8020029

Academic Editor: Fabrizio Bertelloni

Received: 18 January 2021

Accepted: 15 February 2021

Published: 17 February 2021

Publisher's Note: MDPI stays neutral with regard to jurisdictional claims in published maps and institutional affiliations.

\section{Copyright: (c) 2021 by the authors.} Licensee MDPI, Basel, Switzerland. This article is an open access article distributed under the terms and conditions of the Creative Commons Attribution (CC BY) license (https:// creativecommons.org/licenses/by/ $4.0 /)$.

\begin{abstract}
The Gram-negative bacterium Riemerella anatipestifer (RA) is known to cause clinical disease with severe economic impacts primarily in ducks and less frequently in geese and turkeys. RA was isolated and identified in broiler chickens, from a clinical case in a commercial broiler farm located in the southwest mainland of Greece. The morbidity and the mortality in the broiler house were estimated at $10 \%$ and $5 \%$ respectively. The observed clinical signs appeared at the age of 30 to 42 days with respiratory distress (dyspnea), white fluid feces and stunting. Post-mortem examinations displayed serositis, pericarditis, perihepatitis and airsacculitis. Edematous swelling around the tibio-tarsal joints was observed in some birds. Tissue samples from lesions were streaked on selective media. Three bacterial isolates were identified by matrix-assisted laser desorption/ionization timeof-flight mass spectrometry (MALDI-TOF MS). Moreover, an antibiogram analysis was performed for the three RA strains, using a pattern of 16 common antibiotics to advocate the most effective drugs for a proper treatment. All the RA isolates were sensitive to ceftiofur, sulphamethoxazoletrimethoprim and amoxicillin, whereas all were resistant to gentamicin, tylosin, tetracyclin, colistin sulphate, spectinomycin, lincomycin and oxytetracycline.
\end{abstract}

Keywords: Riemerella anatipestifer; broiler chickens; MALDI-TOF; antibiotic resistance; clinical disease; bacteria

\section{Introduction}

Riemerella anatipestifer (RA) is the aetiologic agent for a serious contagious bacterial disease of the avian species that causes substantial economic losses in commercially important poultry worldwide [1]. RA is a Gram-negative, non-motile, non-spore forming and rod-shaped bacterium belonging to the family Flavobacteriaceae of the phylum Bacteroidetes [2]. The disease occurs worldwide as an acute or chronic septicemia and has been recognized as a prominent problem in countries that have intensive duck production [3].

Outbreaks of RA have been reported for domestic ducks and turkeys, the first ones resulting in a high mortality rate of up to $75 \%$ and a morbidity that is usually as high as $100 \%$ [1,4-6]. Furthermore, within the wild birds, some cases of the disease have also been mentioned $[7,8]$. In general, affected birds have displayed clinical symptoms such as ocular and nasal discharges, mild coughing and sneezing, tremors of the head and neck, joints' inflammation causing a paddling movement of the legs. Post-mortem lesions were commonly characterized by fibrinous polyserositis, pericarditis, perihepatitis, airsacculitis and in chronic cases by salpingitis and meningitis [9]. 
The present study describes an identified outbreak of RA disease in broiler chickens in Greece, including clinical signs and post-mortem lesions. For the first time, three clinical isolates of RA in broiler chickens were identified and differentiated by matrix-assisted laser desorption ionization-time-of-flight mass spectrometry (MALDI-TOF MS). In addition, the antimicrobial sensitivity of the isolated RA strains was examined, in order to advocate the best drugs for treatment.

\section{Materials and Methods}

\subsection{Ethical Statement}

All birds used in this study were collected by veterinarians from a clinical case of disease in a commercial broiler chicken farm. These birds were humanely euthanized due to severe clinical illness and stunted growth and submitted to post-mortem examination according to national regulations.

\subsection{Selections of Cases}

In October 2019, a possible outbreak of a contagious respiratory disease was reported in single house with 20,000 broilers, from a commercial broiler chicken farm with a total capacity for 70,000 broilers, located in the southwest area of Greece. Based on the farm data, the morbidity in this broiler house was estimated at approximately $10 \%$ and mortality at $5 \%$. Under veterinary supervision, a combination of doxycycline, tylosin and colistin was administered to the birds via drinking water as blank treatment, since the symptoms were suggestive of colibacillosis.

From this farm, six live birds (40-days-old, Ross 308) were submitted for examination to the Laboratory of Animal Health-Food Hygiene and Quality of the School of Agriculture, University of Ioannina. All birds were characterized by stunted growth, estimated $30-40 \%$ lower weight, in comparison to the average flock weight as well as to the target weights for their age [10]. Clinical examination showed that out of the six birds, five were presenting clinical symptoms such as respiratory distress (dyspnea) and white fluid feces.

\subsection{Post-Mortem Examination}

Post-mortem examination was performed to evaluate the severity of organ damage. From all six birds, tissue samples were collected from viscera with profound lesions, including liver, air sacs and pericardium.

\subsection{Riemerella Anatipestifer Isolation and Identification}

All tissue samples were immediately streaked on Columbia sheep blood (CSB) agar, containing $5 \%$ sheep blood, and also on McConkey agar.

Initially, CSB were incubated aerobically for $24 \mathrm{~h}$ at $37^{\circ} \mathrm{C}$. However, after the identification of RA by the MALDI-TOF MS (as described below) in some CSB agars, sub cultured colonies were taken and streaked again in CSB agars and incubated for $24 \mathrm{~h}$ at $37^{\circ} \mathrm{C}$ under microaerophilic conditions $\left(5 \% \mathrm{CO}_{2}\right)$. The same samples that have been inoculated on McConkey agar plates and incubated aerobically at $37^{\circ} \mathrm{C}$, did not show any bacterial growth 24 or $48 \mathrm{~h}$ later.

The phenotypic identification of the isolates was based on their morphology, culture characteristics and biochemical tests. The isolates showed non hemolytic zone on blood agar plate. Preliminary identification was based on phenotypic characteristics (growth, shape, motility). Smears were stained with Gram's stain according to standard techniques. Gram's staining revealed the presence of single or paired Gram-negative short rods. In addition, catalase, oxidase and gelatinase tests were performed [11-13]. The physiologic and biochemical properties were compared to those of type strain ATCC 11845. All agars and reagents were procured from Merck KGaA (Darmstadt, Germany). 
2.5. Sample Preparation for MALDI-TOF MS Identification: Parameters and Main Spectral Profile (MSP) Dendrogram Construction

The identification of RA isolates was conducted using the mass spectrometer Microflex LT MALDI-TOF MS (Bruker Daltonic, Bremen, Germany). A total of 240 laser shots in 40 shot steps were summarized and each spot was measured twice automatically with AutoXecute acquisition control software (flexControl 3.4; Bruker Daltonic, Bremen, Germany). Ethanol-formic acid-acetonitrile standard operation protocol, was evaluated in order to achieve a complete protein extraction [14]. The reference library from Bruker Daltonic was used to identify the isolates. The instrument was calibrated using a Bacterial Test Standard (BTS-containing a typical Escherichia coli DH5 alpha peptide and protein profile plus additional proteins) (Bruker Daltonic, Bremen, Germany). The generated peak list was matched against the database using the pattern-matching algorithm of MALDI Biotyper software version 3.4 (Bruker Daltonic, Bremen, Germany). Twenty four mass spectra of each RA strain were obtained and were converted into master spectra (MSPs). Five RA reference strains databank entries were included as controls (MALDI Biotyper 3.4, Bruker Daltonic, Bremen, Germany).

\subsection{Antibiotic Sensitivity Test}

Antibiotic sensitivity pattern of three RA isolates was carried out using the standard Kirby-Bauer disk diffusion method [15]. Sixteen antibiotics were used to determine the antibiotic susceptibility of RA isolates, including: $25 \mu \mathrm{g}$ of Amoxicillin (AMOXY25, Oxoid Basingstoke, Hampshire, UK), $10 \mu \mathrm{g}$ of Ampicillin (AMP10, Oxoid, Basingstoke, Hampshire, UK), $30 \mu \mathrm{g}$ of Ceftiofur (CFT30, Rosco Diagnostica, Taasrup, Denmark), $30 \mu \mathrm{g}$ of Ciprofloxacin (CIP30, Oxoid, Basingstoke, Hampshire, UK), $10 \mu \mathrm{g}$ of Colistin sulfate (CT10, Oxoid, Basingstoke, Hampshire, UK), $30 \mu \mathrm{g}$ of Doxycycline (DOXYC30, Rosco Diagnostica, Taasrup, Denmark), $5 \mu \mathrm{g}$ of Enrofloxacin (ENR5, Oxoid, Basingstoke, Hampshire, UK), $10 \mu \mathrm{g}$ of Gentamicin (CN10, Oxoid, Basingstoke, Hampshire, UK), $15 \mu \mathrm{g}$ of Lincomycin (MY15, Oxoid, Basingstoke, Hampshire, UK), $10 \mu \mathrm{g}$ of Neomycin (N10, Oxoid, Basingstoke, Hampshire, UK), $30 \mu \mathrm{g}$ of Oxytetracycline (OT30, Sanofi Diagnostics Pasteur, Marnes-la-Coquette, France), $10 \mu \mathrm{g}$ of Penicillin G (P10, Oxoid, Basingstoke, Hampshire, UK), $100 \mu \mathrm{g}$ of Spectinomycin (SH100, Oxoid, Basingstoke, Hampshire, UK), $25 \mu \mathrm{g}$ of Sulphamethoxazole-Trimethoprim (SXT25, Oxoid, Basingstoke, Hampshire, UK), $30 \mu \mathrm{g}$ of Tetracyclin (TE30, Oxoid, Basingstoke, Hampshire, UK) and $30 \mu \mathrm{g}$ of Tylosin (TY30, Liofilchem, Teramo, Italy). Disk diffusion analysis was performed on Mueller-Hinton agar enriched with $5 \%$ defibrinated sheep blood and was incubated at $37^{\circ} \mathrm{C}$ in microaerophilic atmosphere with 5\% $\mathrm{CO}_{2}$. Quality control isolates included Escherichia coli ATCC 25,922 and Staphylococcus aureus ATCC 25923. Inhibitory zone diameters were measured after $24 \mathrm{~h}$ of incubation.

The resistance breakpoints were interpreted according to the criteria provided by Clinical and Laboratory Standards Institute (CLSI) documents M100-S21 and VET01S [16,17], of the National Food Chain Safety Office, Veterinary Diagnostic Directorate. The interpretation was based on CLSI document VET01 fifth edition [17], as well as described in previous reports $[18,19]$.

\section{Results}

\subsection{Post-Mortem Findings}

Post-mortem lesions were observed in five of the six examined birds. The examinations showed airsacculitis, serositis, pericarditis and perihepatitis (Figure 1A-C). Edematous swelling around the tibio-tarsal joints was observed in three out of the six birds. 


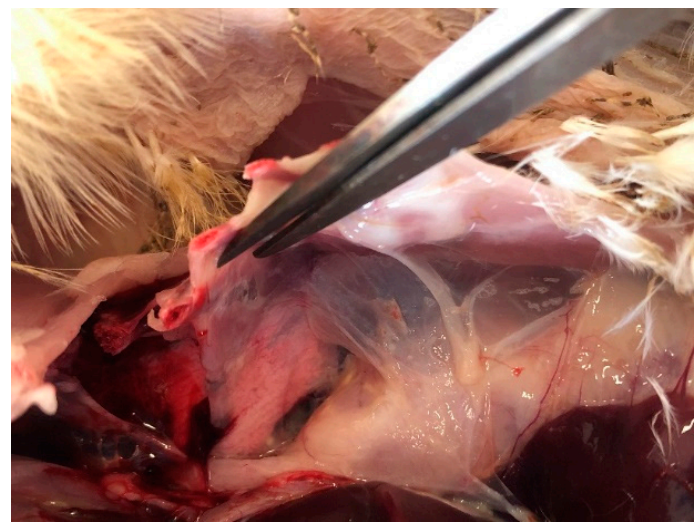

(a)

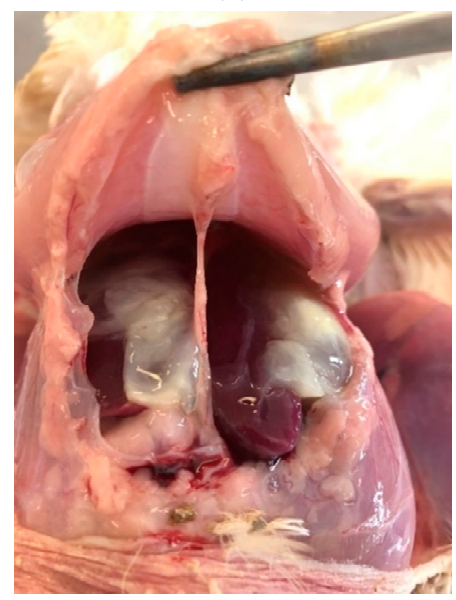

(b)

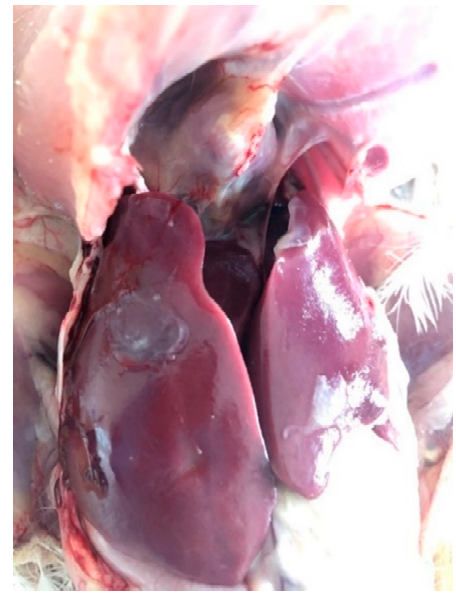

(c)

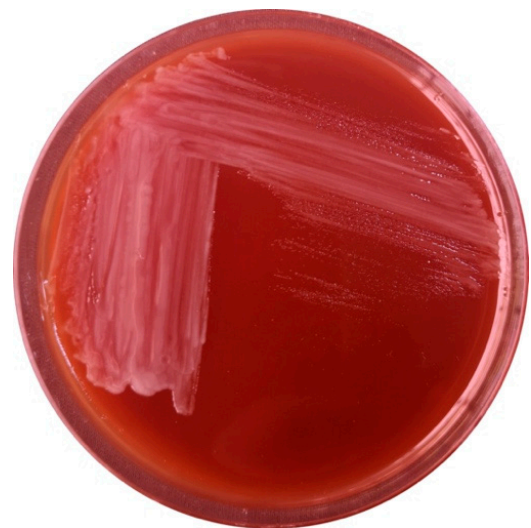

(d)

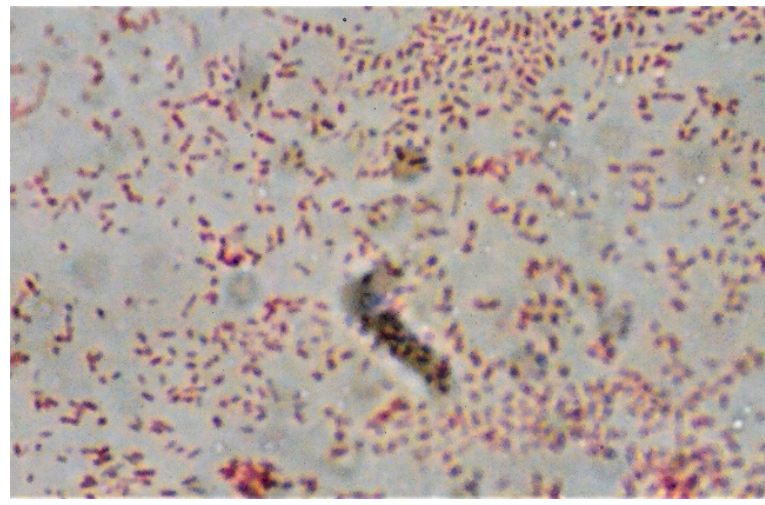

(e)

Figure 1. Riemerella anatipestifer: (a) Airsacculitis of the thoracic air sacs in broiler chicken filled with organized yellow casts; (b) Serositis in broiler chicken; (c) Pericarditis \& perihepatitis in broiler chicken; (d) Transparent, glistening and non-hemolytic colonies on Sheep Blood Agar (CBS); (e) Gram-stained smear from culture showing the typical pleomorphic appearance. 


\subsection{Isolation and Identification of Riemerella anatipestifer}

Streaked organ samples on CSB agar were positive for RA in two out of five affected birds. Positive samples were designated as: RA1 (Sample from air sac from bird No.3), RA2 (Sample from liver from bird No.5) and RA3 (Sample from pericardium from bird No.5). The results of the biochemical tests for RA as shown in Table 1. Catalase Oxidase and Gelatinase tests were positive of all three isolates. The physiologic and biochemical properties were similar to those of type strain ATCC 11845.

Typical individual colonies of each isolate were picked for MALDI-TOF analysis. These isolates were identified as RA by MALDI-TOF MS, using the reference database (Table 1). The log score of RA2 (2.15) indicating "secure genus identification, probable species identification," whereas the log scores of RA1 (2.30) and RA3 (2.35) indicating "high probable species identification," based on the reference database guidelines.

Table 1. Identification of Riemerella anatipestifer from broiler chicken tissues using culture, staining and biochemical tests and matrix-assisted laser desorption/ionization time-of-flight mass spectrometry (MALDI-TOF MS) analysis based on the Bruker Daltonic database.

\begin{tabular}{|c|c|c|c|}
\hline Culture Technique and Biochemical Tests & $\begin{array}{c}\text { RA1 } \\
\text { (Bird No.3- } \\
\text { Air Sac) }\end{array}$ & $\begin{array}{c}\text { RA2 } \\
\text { (Bird No.5- } \\
\text { Liver) }\end{array}$ & $\begin{array}{c}\text { RA3 } \\
\text { (Bird No.5- } \\
\text { Pericardium) }\end{array}$ \\
\hline Growth on Columbia sheep blood (CSB) agar aerobically & + & + & + \\
\hline Hemolysis on CSB agar & - & - & - \\
\hline Growth on CSB microaerophilically & + & + & + \\
\hline Growth on MacConkey agar & - & - & - \\
\hline Gram's reaction & - & - & - \\
\hline Catalase test & + & + & + \\
\hline Oxidase test & + & + & + \\
\hline Gelatinase test & + & + & + \\
\hline \multicolumn{4}{|l|}{ MALDI-TOF MS analysis } \\
\hline Matched pattern on the Bruker Daltonic database & R. anatipestifer GD 47 & R. anatipestifer GD & R. anatipestifer GD 47 \\
\hline Log (score) value * & $\begin{array}{c}\text { GDD } \\
2.30\end{array}$ & $\begin{array}{l}\text { 47 GDD } \\
2.15\end{array}$ & $\begin{array}{l}\text { GDD } \\
2.35\end{array}$ \\
\hline
\end{tabular}

* According to MALDI Biotyper software version 3.4 (Bruker Daltonic, Bremen, Germany) a log score value between 2.0-3.0 corresponds to

"high confidence species identification".

Although RA isolates (RA1, RA2 and RA3) were grown under aerobic conditions on CSB agar initially, the growth of these bacteria was optimal in the same medium under an atmosphere of $5 \% \mathrm{CO}_{2}$. Colonies were small, 1-2 $\mathrm{mm}$ in diameter, transparent, glistening and non-hemolytic (Figure 1D). There was no growth on MacConkey agar. Microscopic observation of CSB agar colonies revealed short, Gram-staining-negative, non-sporulating, rod-shaped bacteria (Figure 1E).

For the construction of the MSP dendrogram based on the results from MALDI-TOF MS (Figure 2A), the data were processed with default software settings. The resulting MSP dendrogram (Figure 2B) shows that the RA2 and RA3 isolates of RA are closely related (distance level < 100) and clustered with the RA1 strain (distance level < 200) to the species level [20]. 


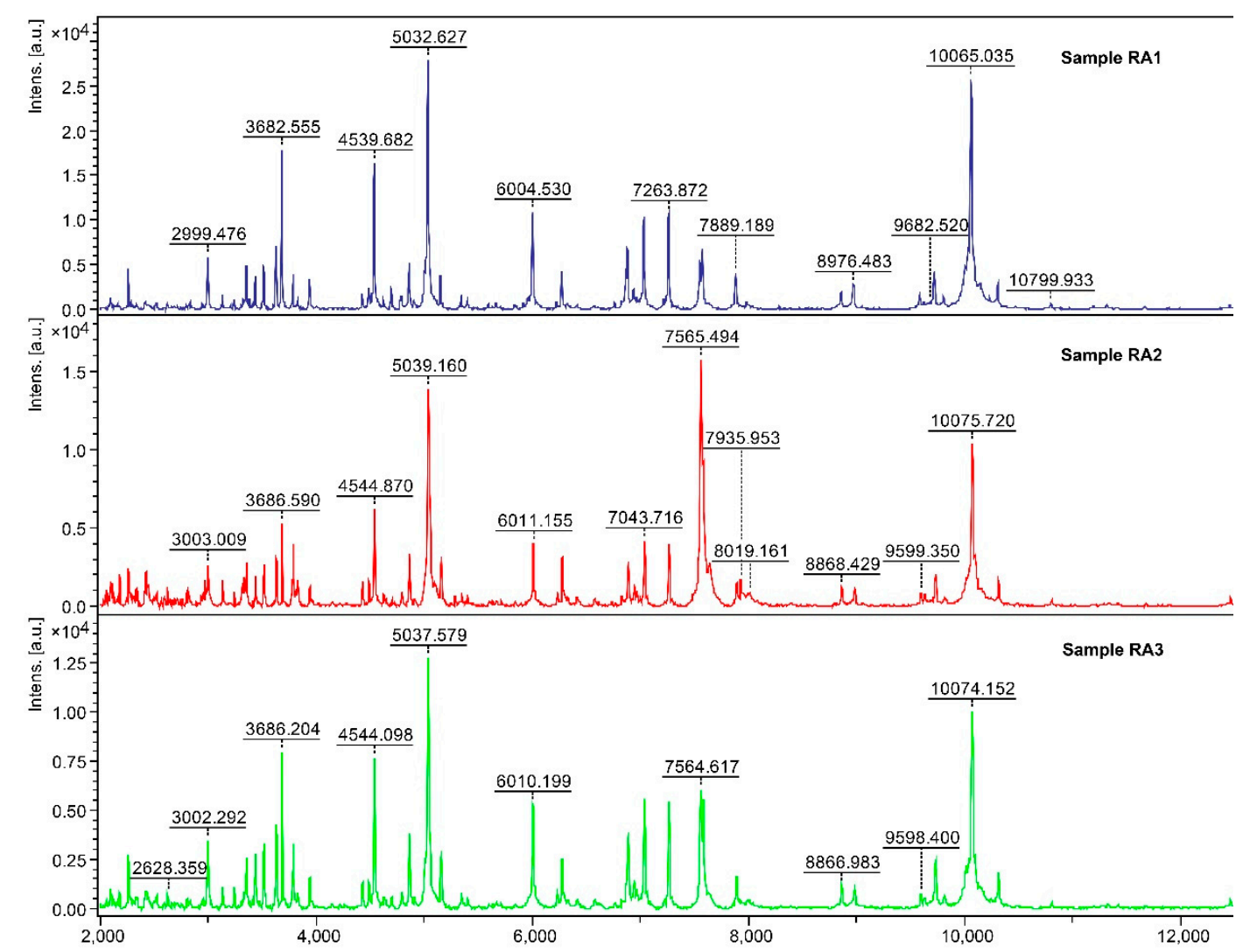

(a)

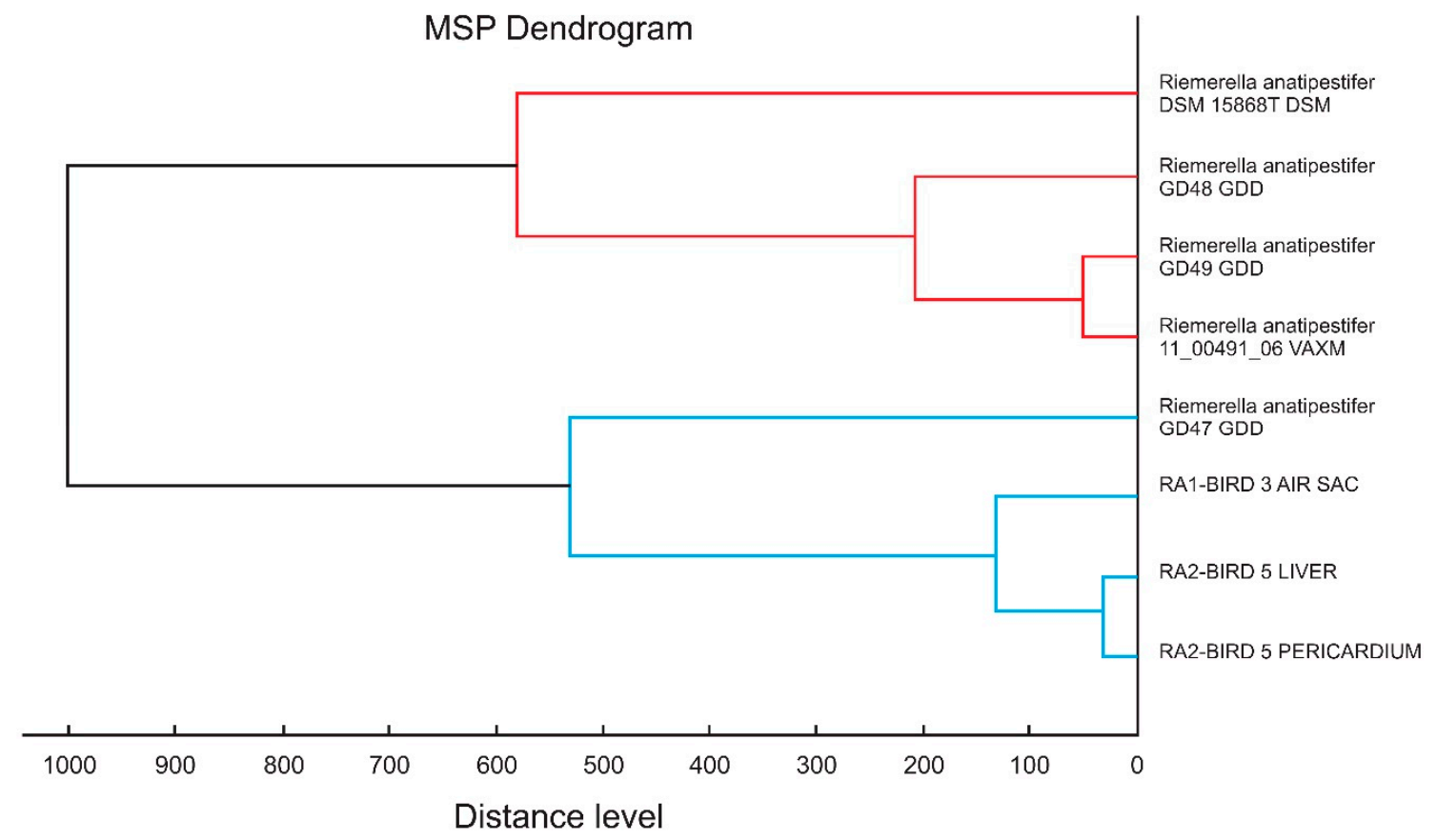

(b)

Figure 2. (a) Mass spectra for Riemerella anatipestifer (RA) isolates, analyzed by MALDI-TOF MS; (b) MSP dendrogram: Classification of three isolates of Riemerella anatipestifer, based on the protein mass patterns, analyzed by MALDI Biotyper software using MALDI-TOF MS. 


\subsection{Antibiotic Susceptibility Testing Results}

Table 2 presents the antibiotic sensitivity pattern of RA isolates and the resistance breakpoints of the antibiotics used in this study. The three RA isolates displayed a clear sensitivity profile to three antibiotics, which were ceftiofur, sulphamethoxazole-trimethoprim and amoxicillin. All the RA isolates displayed an expanding resistance pattern to gentamicin, tylosin, tetracyclin, colistin sulfate, spectinomycin, lincomycin and oxytetracycline. Other antibiotics which were tested such as ampicillin, penicillin, enrofloxacin, neomycin, ciprofloxacin and doxycycline, showed variable resistance and sensitivity pattern between the clinical RA isolates.

Table 2. Resistance breakpoints and antibiotic sensitivity pattern of Riemerella anatipestifer from broiler chicken tissues.

\begin{tabular}{|c|c|c|c|c|c|c|}
\hline & \multicolumn{3}{|c|}{$\begin{array}{l}\text { Interpretive Criteria: } \\
\text { Zone Diameter }(\mathrm{mm})\end{array}$} & \multirow{2}{*}{$\begin{array}{c}\text { RA1 } \\
\text { (Bird No.3- } \\
\text { Air Sac) }\end{array}$} & \multirow{2}{*}{$\begin{array}{c}\text { RA2 } \\
\text { (Bird No.5- } \\
\text { Liver) }\end{array}$} & \multirow{2}{*}{$\begin{array}{c}\text { RA3 } \\
\text { (Bird No.5- } \\
\text { Pericardium) }\end{array}$} \\
\hline & Resistant & Intermediate & Susceptible & & & \\
\hline Amoxycillin $(25 \mu \mathrm{g})$ & $\leq 18$ & $19-20$ & $\geq 21$ & Sensitive & Sensitive & Sensitive \\
\hline Ampicillin $(10 \mu \mathrm{g})$ & $\leq 23$ & & $\geq 24$ & Resistant & Resistant & Sensitive \\
\hline Ceftiofur $(30 \mu \mathrm{g})$ & $<18$ & & $\geq 21$ & Sensitive & Sensitive & Sensitive \\
\hline Ciprofloxacin $(30 \mu \mathrm{g})$ & $\leq 15$ & $16-20$ & $\geq 21$ & Sensitive & Sensitive & Intermediate \\
\hline Colistin Sulphate $(10 \mu \mathrm{g})$ & $\overline{\leq} 16$ & $17-19$ & $\geq 20$ & Resistant & Resistant & Resistant \\
\hline Doxycycline $(30 \mu \mathrm{g})$ & $<22$ & & $\geq 23$ & Resistant & Sensitive & Sensitive \\
\hline Enrofloxacin $(5 \mu \mathrm{g})$ & $\leq 16$ & $17-22$ & $\geq 23$ & Resistant & Resistant & Sensitive \\
\hline Gentamicin $(10 \mu \mathrm{g})$ & $\leq 12$ & $13-14$ & $\geq 15$ & Resistant & Resistant & Resistant \\
\hline Lincomycin $(15 \mu \mathrm{g})$ & $<17$ & & $\geq 21$ & Resistant & Resistant & Resistant \\
\hline Neomycin $(10 \mu \mathrm{g})$ & $\leq 16$ & & $\geq 17$ & Resistant & Sensitive & Resistant \\
\hline Oxytetracycline $(30 \mu \mathrm{g})$ & $<17$ & & $\geq 19$ & Resistant & Resistant & Resistant \\
\hline Penicillin G $(10 \mu \mathrm{g})$ & $\leq 23$ & & $\geq 24$ & Sensitive & Sensitive & Resistant \\
\hline Spectinomycin $(100 \mu \mathrm{g})$ & $\leq 15$ & 16 & $\geq 17$ & Resistant & Resistant & Resistant \\
\hline $\begin{array}{l}\text { Sulphamethoxazole-Trimethoprim } \\
\text { SXT }(25 \mu \mathrm{g})\end{array}$ & $\leq 10$ & $11-15$ & $\geq 16$ & Sensitive & Sensitive & Sensitive \\
\hline Tetracycline $(30 \mu \mathrm{g})$ & $\leq 22$ & & $\geq 23$ & Resistant & Resistant & Resistant \\
\hline Tylosin $(30 \mu \mathrm{g})$ & $<14$ & & $\geq 18$ & Resistant & Resistant & Resistant \\
\hline
\end{tabular}

\section{Discussion}

Our results document a detected clinical case of an infection caused by RA in a commercial poultry farm in Greece. Based on available published literature, for the first time, in our case report, clinical RA isolates originating from broiler chickens were identified at species level, by MALDI-TOF MS.

In the published literature clinical cases of RA in chickens are scarce. Rosenfeld [21] reported that RA had been isolated and identified in chickens with atypical signs. Since then, a field outbreak of RA disease in chickens has also been described in China [22].

In our clinical and post-mortem findings, the observed post-mortem gross lesions included fibrinous exudate in the pericardial cavity and over the surface of the liver, as well as airsacculitis filled with organized yellow casts, all of which are characteristic findings reported in avian species [8,23-25].

Standard methods of isolating microorganisms in suitable agar media were initially used, with samples taken from post-mortem lesions from the pericardium, airsacs and liver. Out of all samples from six birds, RA growth was detected only in three plated organ samples from two birds. We identified these colonies as RA using the MALDI-TOF MS, allowing us to continue their subcultures under microaerophilic conditions with much better growth. At the same time, no other of the possible examined pathogenic bacteria like E. coli and Salmonella spp. were grown from the same tissues. These observations are in line with previous reports in geese [23].

Diagnosis of RA infection is difficult, using standard microbiological procedures $[13,26]$. Particularly in our case both CSB and MacConkey agar were inoculated from the lesions found in the examined birds. RA colonies were grown only on the CSB agar, confirming 
the existence of this bacterium. Additionally, the microaerophilic conditions during the second cultivation promoted the growth of RA and revealed its existence. Polymerase chain reaction (PCR) methods have been used by many studies to identify the isolates obtained but most of the PCR assays designed for RA detection, are proven to be ineffective to detect or specify all RA strains $[27,28]$. The use of a MALDI Biotyper is considered to give more reliable results for RA identification, making use of well-described RA reference strains $[23,26,29]$, while being adequately simple and cost-effective for routine laboratory use [23]. RA have been successfully isolated and identified with MALDI-TOF MS from clinical cases from ducks, goose and turkeys, as previously described [23,26,29].

In our study, clinical RA isolates originating from broiler chickens were identified at species level, by MALDI-TOF MS. The protein profiling, via MALDI-TOF MS, of the three RA clinical isolates indicated that the two RA isolates from different tissues of the same bird (RA2-liver \& RA3-pericardium of bird No. 5) are clustered together with high affinity (distance level $<100$ ) and subclustered with the RA1 isolate from the other infected bird (RA1-airsac of bird No. 3) (distance level < 200) indicating high correlation. All the Bruker databank entries of RA, except from the databank entry "Riemerella Anatispetifer 11-00491-06 VAXM," have been derived from ducks. Our three RA clinical isolates derived from chickens, are not reliable related (distance level > 500) [20], with these RA Bruker databank entries (including the RA reference type strain (DSM 15868T R). In the resulting MSP dendrogram we observed that the RA clinical isolates have been reliably classified to the same species and to different subspecies, which demonstrates the existence of the genetic variation into RA strains [30].

The large genetic diversity of the RA strains with the poor cross-protection between them discourages the prevention using vaccines, making antimicrobial therapy the primary method of fighting RA infection [31]. Thus, in vitro drug sensitivity testing is essential for the selection of an appropriate antibiotic for a given situation.

The severity of RA infections in the avian breeding industry, has mainly been controlled by the wide use of quinolones, tetracyclines and cephalosporins, which have consequently led to the emergence of antibiotic-resistant strains specifically in ducks [32]. Likewise, with many other bacterial pathogens worldwide, the incidence of drug resistance in the treatment of RA infections is increasing [33,34].

In our study, three RA isolates were tested versus 16 antibiotics, widely used agents in poultry industry, in order to determine the susceptibility in each antimicrobial agent. All three RA isolates displayed a clear sensitivity profile to amoxicillin, ceftiofur and sulphamethoxazole-trimethoprim. Chang et al. [35] reported a high percentage (97.4\%) of the tested RA isolates which were susceptible to amoxicillin. Ceftiofur, is an approved third generation cefalosporin for food animal use in the United States and Europe [36]. Studies in duck isolates in Taiwan also approved that the ceftiofur is among the most effective antibiotics. Chang et al. [35] found that sulphamethoxazole-trimethoprim showed 57\% and 50\% efficacy RA isolates from geese and ducks, respectively. Sun et al. [37] noted that Chinese ducks' RA isolates, were resistant to sulphonamide, while $75 \%$ of the strains were susceptible to sulphamethoxazole-trimethoprim.

According to our results, all the RA isolates displayed an expanding resistance pattern to eight antibiotics: colistin sulfate, spectinomycin, gentamicin, lincomycin, neomycin, oxytetracycline, spectinomycin, tetracycline and tylosin. Chang et al. [35] examined antibiotic resistant of RA in ducks and geese, demonstrated that $50 \%$ or more of the isolates had developed resistance against commonly used antibiotics. Gyuris et al. [19] found that more than one half of the RA isolates from geese and ducks in Hungary had developed resistance against gentamicin. The high prevalence of colistin resistance in the isolates was also reported in previous studies [35,38]. According to the same results, more than $70 \%$ of the isolates were resistant to lincomycin as well [35,39]. Colistin and lincomycin are approved for use in feed treatments for bacterial infections in poultry. Apart from colistin, resistance has also emerged to spectinomycin, which is often used in poultry practice [19]. 
In this study, differences were detected in the sensitivity and resistance pattern between the three RA isolates, in several antibiotics such as ampicillin, penicillin, enrofloxacin, neomycin, ciprofloxacin and doxycycline. Of remarkable note is also the difference in sensitivity and resistance at doxycycline, an antibiotic commonly used in poultry production. In a previous study, conducted by Zhong et al. [18] it was reported that RA isolates were resistant to ampicillin, which is in agreement with our results as two from the three RA isolates were resistant to this drug. Two of the three RA isolates in this study were resistant to enrofloxacin. Sensitivity to enrofloxacin against RA isolated from ducklings was reported by Turbahn et al. [40] and Soman et al. [41].

Regarding the origin of the contamination, our assumption was that the pathogen possibly spread from a duck breeding farm that was adjacent to the broiler farm, where during the same period faced some moderate mortalities from unspecified causes as it was reported by the farmer. Unfortunately, it was not possible to procure samples from these ducks for laboratory analysis. Consequently, it is very important to highlight the need to maintain a high level of biosecurity and good hygiene standards and practices, especially in areas with high numbers of animal and multiple species of reared poultry.

Author Contributions: Conceptualization, A.T. (Athina Tzora) and I.S.; Methodology, A.T. (Athina Tzora), S.S., E.B., K.F., A.K., A.N., A.T. (Anastasios Tsinas) and I.S.; Validation, E.B. and A.T. (Anastasios Tsinas); Format analysis, E.B., I.G. and A.T. (Anastasios Tsinas); Investigation, A.T. (Athina Tzora), S.S., K.F., A.K., A.N., E.B., A.T. (Anastasios Tsinas) and I.S.; Data curation, A.T. (Athina Tzora), E.B., I.G. and A.T. (Anastasios Tsinas); Writing-original draft preparation, A.T. (Athina Tzora), I.S. and E.B.; Writing-overview and editing, A.T. (Athina Tzora), E.B., I.S., I.G. and S.S.; Visualization, A.T. (Athina Tzora) and E.B.; Supervision, A.T. (Athina Tzora), I.S. and E.B.; Project administration, I.S.; Funding acquisition, I.S. All authors have read and agreed to the published version of the manuscript.

Funding: This research has been co-financed by Greece and the European Union (European Regional Development Fund) in context "Research-Create-Innovate" within the Operational Program (Competitiveness, Entrepreneurship and Innovation (EPAnEK) of the NSRF 2014-2020. Project Code: T1EAK-03856. Acronym “GREEN POULTRY MEAT ANTIFREE”.

Institutional Review Board Statement: All birds used in this study were collected by veterinarians from a clinical case of disease in a commercial broiler chicken farm. These birds were humanely euthanized due to severe clinical illness and stunted growth and submitted to post-mortem examination according to national regulations. The study was conducted according to the guidelines of the according to national ethical guidelines and regulations.

Informed Consent Statement: Not applicable.

Data Availability Statement: The data presented in this study are available on request from the corresponding author.

Conflicts of Interest: The authors declare no conflict of interest. The funders had no role in the design of the study; in the collection, analyses, or interpretation of data; in the writing of the manuscript, or in the decision to publish the results.

\section{References}

1. Saif, Y.M.; Fadly, A.M.; Glisson, J.R.; McDougald, L.R.; Nolan, L.K.; Swayne, D.E. Diseases of Poultry, 12th ed.; Blackwell Publishing: Ames, IA, USA, 2008.

2. Segers, P.; Mannheim, W.; Vancanneyt, M.; De Brandt, K.; Hinz, K.H.; Kersters, K.; Vandamme, P. Riemerella anatipestifer gen. nov., comb. nov., the causative agent of septicemia anserum exsudativa, and its phylogenetic affiliation within the FlavobacteriumCytophaga rRNA homology group. Int. J. Syst. Evol. Microbiol. 1993, 43, 768-776. [CrossRef]

3. Pathanasophon, P.; Phuektes, P.; Tanticharoenyos, T.; Narongsak, W.; Sawada, T. A potential new serotype of Riemerella anatipestifer isolated from ducks in Thailand. Avian Pathol. 2002, 31, 267-270. [CrossRef]

4. Cooper, G.L. Pasteurella anatipestifer infections in California turkey flocks: Circumstantial evidence of a mosquito vector. Avian Dis. 1989, 33, 809-815. [CrossRef]

5. Fulton, R.M.; Rimler, R.B. Epidemiologic investigation of Riemerella anatipestifer in a commercial duck company by serotyping and DNA fingerprinting. Avian Dis. 2010, 54, 969-972. [CrossRef] [PubMed] 
6. Yu, C.Y.; Liu, Y.W.; Chou, S.J.; Chao, M.R.; Weng, B.C.; Tsay, J.G.; Chiu, C.H.; Wu, C.C.; Lin, T.S.; Chang, C.C.; et al. Genomic diversity and molecular differentiation of Riemerella anatipestifer associated with eight outbreaks in five farms. Avian Pathol. 2008, 37, 273-279. [CrossRef]

7. Bruner, D.W.; Angstrom, C.I.; Price, J.I. Pasteurella anatipestifer infection in pheasants. A case report. Cornell. Vet. 1970, 50, 491-494.

8. Karstad, L.; Lusis, P.; Long, J.R. Pasteurella anatipestifer as a cause of mortality in captive wild waterfowl. J. Wildl. Dis. 1970, 6, 408-413. [CrossRef] [PubMed]

9. Wobeser, G.A. Reimerella anatipestifer infection. In Diseases of Wild Waterfowl, 2nd ed.; Springer Science \& Business Media, LLC: New York, NY, USA, 1997; pp. 78-81.

10. Aviagen. ROSS 308 Broiler: Performance Objectives; Aviagen: Huntsville, AL, USA, 2014.

11. Ryll, M.; Christensen, H.; Bisgaard, M.; Christensen, J.P.; Hinz, K.H.; Köhler, B. Studies on the prevalence of Riemerella anatipestifer in the upper respiratory tract of clinically healthy ducklings and characterization of untypable strains. J. Vet. Med. Ser. B 2001, 48, 537-546. [CrossRef] [PubMed]

12. Rubbenstroth, D.; Martin, R.; Hotzel, H.; Christensen, H.; Knobloch, J.; Rautenschlein, S.; Bisgaard, M. Description of Riemerella columbipharyngis sp. nov., isolated from the pharynx of healthy domestic pigeons (Columba livia f. domestica), and emended descriptions of the genus Riemerella, Riemerella anatipestifer and Riemerella columbina. Int. J. Syst. Evol. Microbiol. 2013, 63, $280-287$. [CrossRef]

13. Markey, B.; Leonard, F.A.; Archambault, M.; Cullinane, A.; Maguire, D. Clinical Veterinary Microbiology; Elsevier Health Sciences: Oxford, UK, 2013.

14. Bastin, B.; Bird, P.; Benzinger, M.J.J.; Crowley, E.; Agin, J.; Goins, D. Confirmation and identification of Salmonella spp., Cronobacter spp., and other Gram-negative organisms by the Bruker MALDI biotyper method: Collaborative study, first action 2017.09. J. AOAC Int. 2018, 101, 1593-1609. [CrossRef]

15. Bauer, A.W. Antibiotic susceptibility testing by a standardized single disc method. Am. J. Clin. Pathol. 1966, 45, 149-158. [CrossRef]

16. CLSI. Performance Standards for Antimicrobial Susceptibility Testing: Twenty-First Informational Supplement-CLSI Document M100-S21; Clinical \& Laboratory Standards Institute: Wayne, PA, USA, 2011.

17. CLSI. Performance Standards for Antimicrobial Disk and Dilution Susceptibility Tests for Bacteria Isolated from Animals, 5th ed.; CLSI Document VET01S; Clinical \& Laboratory Standards Institute: Wayne, PA, USA, 2018.

18. Zhong, C.Y.; Cheng, A.C.; Wang, M.S.; Zhu, D.K.; Luo, Q.H.; Zhong, C.D.; Li, L.; Duan, Z. Antibiotic susceptibility of Riemerella anatipestifer field isolates. Avian Dis. 2009, 53, 601-607. [CrossRef]

19. Gyuris, E.; Wehmann, E.; Czeibert, K.; Magyar, T. Antimicrobial susceptibility of Riemerella anatipestifer strains isolated from geese and ducks in Hungary. Acta Vet. Hung. 2017, 65, 153-165. [CrossRef] [PubMed]

20. Sauer, S.; Freiwald, A.; Maier, T.; Kube, M.; Reinhardt, R.; Kostrzewa, M.; Geider, K. Classification and Identification of bacteria by mass spectrometry and computational analysis. PLOS ONE 2008, 3, e2843. [CrossRef] [PubMed]

21. Rosenfeld, L.E. Pasteurella anatipestifer infection in fowls in Australia. Aust. Vet. J. 1973, 49, 55-56. [CrossRef] [PubMed]

22. Li, J.X.; Tang, Y.; Gao, J.Y.; Huang, C.H.; Ding, M.J. Riemerella anatipestifer infection in chickens. Pak. Vet. J. $2011,31,65-69$.

23. Hess, C.; Enichlmayr, H.; Jandreski-Cvetkovic, D.; Liebhart, D.; Bilic, I.; Hess, M. Riemerella anatipestifer outbreaks in commercial goose flocks and identification of isolates by MALDI-TOF mass spectrometry. Avian Pathol. 2013, 42, 151-156. [CrossRef]

24. Smith, J.M.; Frame, D.D.; Cooper, G.; Bickford, A.A.; Ghazikhanian, G.Y.; Kelly, B.J. Pasteurella anatipestifer infection in commercial meat-type turkeys in California. Avian Dis. 1987, 31, 913-917. [CrossRef] [PubMed]

25. Leibovitz, L. A Survey of the So-Called “Anatipestifer Syndrome”. Avian Dis. 1972, 16, 836-851. [CrossRef]

26. Rubbenstroth, D.; Ryll, M.; Knobloch, J.K.M.; Köhler, B.; Rautenschlein, S. Evaluation of different diagnostic tools for the detection and identification of Riemerella anatipestifer. Avian Pathol. 2013, 42, 17-26. [CrossRef]

27. Christensen, H.; Bisgaard, M. Phylogenetic relationships of Riemerella anatipestifer serovars and related taxa and an evaluation of specific PCR tests reported for R. anatipestifer. J. Appl. Microbiol. 2010, 108, 1612-1619. [CrossRef]

28. Tsai, H.J.; Liu, Y.T.; Tseng, C.S.; Pan, M.J. Genetic variation of the ompA and 16S rRNA genes of Riemerella anatipestifer. Avian Pathol. 2005, 34, 55-64. [CrossRef]

29. Rubbenstroth, D.; Hotzel, H.; Knobloch, J.; Teske, L.; Rautenschlein, S.; Ryll, M. Isolation and characterization of atypical Riemerella columbina strains from pigeons and their differentiation from Riemerella anatipestifer. Vet. Microbiol. 2011, 147, 103-112. [CrossRef]

30. Wang, X.; Liu, W.; Zhu, D.; Yang, L.F.; Liu, M.F.; Yin, S.; Wang, M.S.; Jia, R.Y.; Chen, S.; Sun, K.F. Comparative genomics of Riemerella anatipestifer reveals genetic diversity. BMC Genom. 2014, 15, 479. [CrossRef]

31. Guo, Y.; Hu, D.; Guo, J.; Wang, T.; Xiao, Y.; Wang, X.; Li, S.; Liu, M.; Li, Z.; Bi, D. Riemerella anatipestifer type IX secretion system is required for virulence and gelatinase secretion. Front. Microbiol. 2017, 8, 2553. [CrossRef]

32. Li, T.; Shan, M.; He, J.; Wang, X.; Wang, S.; Tian, M.; Qi, J.; Luo, T.; Shi, Y.; Ding, C. Riemerella anatipestifer M949_0459 gene is responsible for the bacterial resistance to tigecycline. Oncotarget 2017, 8, 96615. [CrossRef] [PubMed]

33. Li, Y.F.; Jiang, H.X.; Xiang, R.; Na, S.; Zhang, Y.N.; Zhao, L.Q.; Peng, G.; Wang, L.Q.; Zeng, Z.L. Effects of two efflux pump inhibitors on the drug susceptibility of Riemerella anatipestifer isolates from China. J. Integr. Agric. 2016, 15, 929-933. [CrossRef]

34. Yang, F.F.; Sun, Y.N.; Li, J.X.; Wang, H.; Zhao, M.J.; Su, J.; Zhang, Z.J.; Liu, H.J.; Jiang, S.J. Detection of aminoglycoside resistance genes in Riemerella anatipestifer isolated from ducks. Vet. Microbiol. 2012, 158, 451. [CrossRef] [PubMed] 
35. Chang, F.F.; Chen, C.C.; Wang, S.H.; Chen, C.L. Epidemiology and antibiogram of Riemerella anatipestifer isolated from waterfowl slaughterhouses in Taiwan. J. Vet. Res. 2019, 63, 79-86. [CrossRef] [PubMed]

36. Hornish, R.E.; Kotarski, S.F. Cephalosporins in veterinary medicine-Ceftiofur use in food animals. Curr. Top. Med. Chem. 2002, 2, 717-731. [CrossRef]

37. Sun, N.; Liu, J.H.; Yang, F.; Lin, D.C.; Li, G.H.; Chen, Z.L.; Zeng, Z.L. Molecular characterization of the antimicrobial resistance of Riemerella anatipestifer isolated from ducks. Vet. Microbiol. 2012, 158, 376-383. [CrossRef] [PubMed]

38. Liu, Y.Y.; Wang, Y.; Walsh, T.R.; Yi, L.X.; Zhang, R.; Spencer, J.; Doi, Y.; Tian, G.; Dong, B.; Huang, X. Emergence of plasmidmediated colistin resistance mechanism MCR-1 in animals and human beings in China: A microbiological and molecular biological study. Lancet Infect. Dis. 2016, 16, 161-168. [CrossRef]

39. Luo, H.Y.; Liu, M.F.; Wang, M.S.; Zhao, X.X.; Jia, R.Y.; Chen, S.; Sun, K.F.; Yang, Q.; Wu, Y.; Chen, X.Y. A novel resistance gene, lnu (H), conferring resistance to lincosamides in Riemerella anatipestifer CH-2. Int. J. Antimicrob. Agents 2018, 51, 136-139. [CrossRef]

40. Turbahn, A.; De Jäckel, S.C.; Greuel, E.; De Jong, A.; Froyman, R.; Kaleta, E.F. Dose response study of enrofloxacin against Riemerella anatipestifer septicaemia in Muscovy and Pekin ducklings. Avian Pathol. 1997, 26, 791-802. [CrossRef] [PubMed]

41. Soman, M.; Nair, S.R.; Mini, M.; Mani, B.K.; Joseph, S. Isolation and polymerase chain reaction-based identification of Riemerella anatipestifer from ducks in Kerala, India. Vet. World 2014, 7, 765-769. [CrossRef] 